\title{
LA INCORPORACIÓN DE LA ALFABETIZACIÓN INFORMACIONAL EN LAS BIBLIOTECAS UNIVERSITARIAS IBEROAMERICANAS. ANÁLISIS COMPARATIVO A PARTIR DE LA INFORMACIÓN DE SUS SITIOS WEB
}

\author{
Alejandro Uribe Tirado* \\ Universidad de Antioquia (Colombia) \\ María Pinto Molina** \\ Universidad de Granada
}

\begin{abstract}
Resumen: El artículo busca identificar desde la información que ofrecen los sitios web de las bibliotecas universitarias de los 22 países iberoamericanos, los posibles niveles de incorporación que desde dichas bibliotecas se están realizando para la formación en ALFIN-COMPINFO. Para esa identificación, se utiliza como metodología el análisis de contenidos web a partir de los sitios web de las bibliotecas universitarias iberoamericanas. Los resultados identifican que de un total de 2136 bibliotecas universitarias iberoamericanas, solo 171 dan cuenta de procesos de formación en ALFIN-COMPINFO (6.2\%). El artículo concluye que desde el contexto iberoamericano, la ALFIN-COMPINFO aún no tiene todo el alcance que se esperaría y que se hace necesario, no obstante, cada vez más hay prestigiosas universidades que están asumiendo el reto de esta formación y que mediante el trabajo colaborativo, el benchmarking y el compartir estrategias y objetos de aprendizaje serán guía para que más universidades iberoamericanas desarrollen esta formación.

Palabras clave: Alfabetización informacional; competencias informacionales; bibliotecas universitarias; Iberoamérica; análisis de contenidos; sitios web.
\end{abstract}

Title: INCORPORATING INFORMATION LITERACY IN IBERO-AMERICAN UNIVERSITY LIBRARIES. COMPARATIVE ANALYSIS OF THE INFORMATION FROM THEIR WEBSITES.

Abstracts: This article seeks to identify from the information provided by the websites of university libraries in the 22 Ibero-American countries, the possible levels of integration since these libraries are doing for training in information literacy. The results identify that a total of 2136 Ibero-American university libraries; only 171 have information literacy training programs $(6.2 \%)$. The paper evidences that from the Ibero-American context, information literacy is not yet the full extent you would expect and it is necessary. However, increasingly, there are prestigious universities who are taking on the challenge of this training and therefore, by working collaborative, benchmarking and sharing strategies and learning objects will be a guide for most Ibero-American universities develops this training.

Keywords: Information literacy; information competencies; university libraries; Ibero-America; content analysis; web sites.

\section{INTRODUCCIÓN.}

La alfabetización informacional -ALFIN- o la formación en competencias informacionales -COMPINFO- es una temática que ha venido desarrollándose con diferentes niveles, alcances y trayectorias en Iberoamérica desde el contexto de las bibliotecas, especialmente desde las de nivel universitario.

Como se ha indicado en los textos previos a este trabajo, estos más de 25 años de precisiones y adaptaciones que han llevado al paso de la instrucción bibliográfica y la formación de usuarios tradicional hasta llegar hoy al paradigma de la formación en ALFIN-COMPINFO, se han dado diferentes procesos de avance, períodos de desarrollo (UribeTirado, 2010a-b): «Pre-Inicio (1985-1994); Inicio (1995-1999); Pre-Avance (2000-2003); Avance (2004-2007) у PrePosicionamiento $(2008$ - ...), además de la visualización futura, según se vayan dando determinados procesos y resultados de éxito, de un $6^{\circ}$ período: Posicionamiento (2012, 2013 o 2014...)»; todo esto teniendo en cuenta la integración de la visión y los recuentos históricos realizados por diferentes autores ${ }^{1}$.

Considerando estos procesos-períodos generales, un aspecto clave, un reto, es identificar de manera específica cómo se están llevando a cabo al interior de cada universidad, de cada Institución de Educación Superior (IES) la incorporación de esta formación en ALFIN-COMPINFO, para con ello, poder identificar lecciones aprendidas y

\footnotetext{
*auribe@bibliotecologia.udea.edu.co

mpinto@ugr.es
}

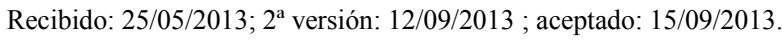

URIBE TIRADO, A. La incorporación de la alfabetización informacional en las bibliotecas universitarias iberoamericanas. Análisis comparativo a partir de la información de sus sitios web. Anales de Documentación, 2013, vol. $16, \mathrm{n}^{\circ} 2$. Disponible en: http://dx.doi.org/10.6018/analesdoc.16.2.175541 
buenas prácticas que permitan a otras universidades-IES de nuestro contexto iberoamericano, con sus debidas adaptaciones, poder mejorar y avanzar en sus programas de formación en competencias informacionales.

Para dicha identificación, una fuente de información clave son los sitios web de las bibliotecas universitarias, ya que especialmente desde la última década, es sin duda este medio, el que más impacto está teniendo en la divulgación e interacción de sus servicios de información-documentación (Detlor y Lewis, 2006; Gardner; Juricek y Xu, 2008; entre otros), donde está incluida la formación.

Específicamente con los servicios de formación (Ferreira-Gomes; Prudêncio y da Conceiçã, 2010), por la divulgación e interacción que permiten en dos vías:

- para los públicos internos: estudiantes, profesores, investigadores y/o directivos de su institución a quienes van dirigidos sus cursos-tutoriales de formación en ALFIN-COMPINFO.

- para los públicos externos: bibliotecólogos, bibliotecarios, profesores y/o directivos de otras IES que quieren conocer la oferta formativa de esa institución y su biblioteca-CRAI para realizar procesos de benchmarking, de trabajo cooperativo interinstitucional, de consorcios formativos, etc.

Por tanto, este texto producto de la investigación «Lecciones aprendidas en programas de alfabetización informacional en universidades de Iberoamérica», que recopila e integra los trabajos específicos para cada país, publicados para los casos de: Argentina, Brasil, Chile, Colombia, Costa Rica, Cuba, España, México, Perú, Portugal, Puerto Rico y Venezuela; o como datos internos de investigación, para los casos de: Bolivia, Ecuador, Guatemala, El Salvador, Honduras, Nicaragua, Panamá, Paraguay y República Dominicana.

El mismo presenta entonces un análisis del caso iberoamericano en general, considerando el análisis de los sitios web de las bibliotecas de sus universidades-IES, y según la información que presentan, identificando los niveles en que se encontrarían en la incorporación de ALFIN-COMPINFO desde una perspectiva de universidad-IES alfabetizada informacionalmente (Webber y Johnston, 2006).

Por tanto, desde nuestra perspectiva, retomando trabajos previos (Uribe Tirado, 2010-2011) que recopilan propuestas de diferentes autores, una universidad-IES o unidad de la misma que deba liderar procesos-programas de formación en ALFIN-COMPINFO (Sistema de Bibliotecas, Biblioteca, CRAI, etc.) puede categorizarse, haciendo una generalización de los distintos contextos, en 4 categorías según el grado de incorporación: universidades-IES comprometidas / en crecimiento / iniciando / desconocedoras.

Estos grados de incorporación de la formación en ALFIN-COMPINFO pueden sintetizarse e identificarse cuando se analizan los sitios web de las bibliotecas de las universidades-IES teniendo en cuenta diferentes elementos (variables), que se resumen en:

Se detecta en el sitio web una sección o información concreta sobre la formación en ALFIN-COMPINFO: (Sí/No: Si la respuesta es No, conllevaría directamente a la categoría de Desconocedora; si la respuesta es SÍ, conllevaría al análisis y clasificación en las categorías según la información que presentan).

7 macrovariables a valorar:

- Definición del programa: Misión, Visión, Objetivos, Plan operativo, Plan pedagógico

- Fundamentación del programa: Definición de ALFIN-COMPINFO, Modelo pedagógico-didáctico de ALFINCOMPINFO y Estándar-indicadores de ALFIN-COMPINFO asumidos

- Estructuración del programa: Cursos ofrecidos, Modalidad de los Cursos, Incorporación de las TIC, Web 2.0 y de Ambientes Virtuales de Aprendizaje, Presencia curricular o extracurricular de los cursos

- Evaluación del programa: Indicadores de gestión, Indicadores de resultados de aprendizaje, Procesos de mejoramiento continuo

Además de otra información complementaria, como:

- Publicaciones sobre este tema y la experiencia de la biblioteca y del programa de ALFIN-COMPINFO: artículos, ponencias, recursos Web 2.0

- Participación en grupos y redes de trabajo sobre esta temática: redes de bibliotecas universitarias, colectivos de bibliotecólogos/bibliotecarios, etc.

- Participación en medios y recursos de divulgación y aprendizaje de esta temática: listas de discusión, blogs, wikis, twitters, boletines, comunidades virtuales, etc.

De estas 7 variables, es especialmente la referente a Estructuración del programa la que puede dar más cuenta del grado de incorporación que ALFIN-COMPINFO tendría en una universidad-IES y su dependencia a cargo (Sistema de Bibliotecas, Biblioteca, CRAI, etc.), ya que la presencia de determinados cursos-tutoriales permite visualizar con 
mayor facilidad el alcance que tiene el programa (facultades, titulaciones, número de estudiantes, etc.) y si se trabajan las 5, 6 o 7 competencias que presentan los modelos-estándares universitarios de ALFIN-COMPINFO (según el que se haya elegido seguir).

Esto conlleva a una correlación de esta variable específica, que se puede visualizar desde los sitios web, con las 4 categorías de incorporación que dan cuenta del proceso de desarrollo de esta temática, que han sido avaladas por expertos internacionales (Sonntag, 2011) y comparte la visión de documentos y propuestas como las de Wang (2007) y de EMPATIC de la Unión Europea (2009) -que cita al marco australiano-neozelandés / ANZIL-, considerando el paso al interior de las universidades-IES de la instrucción bibliográfica y a la formación de usuarios tradicional hasta llegar hoy a la ALFIN-COMPINFO en lo curricular (Figura 1).

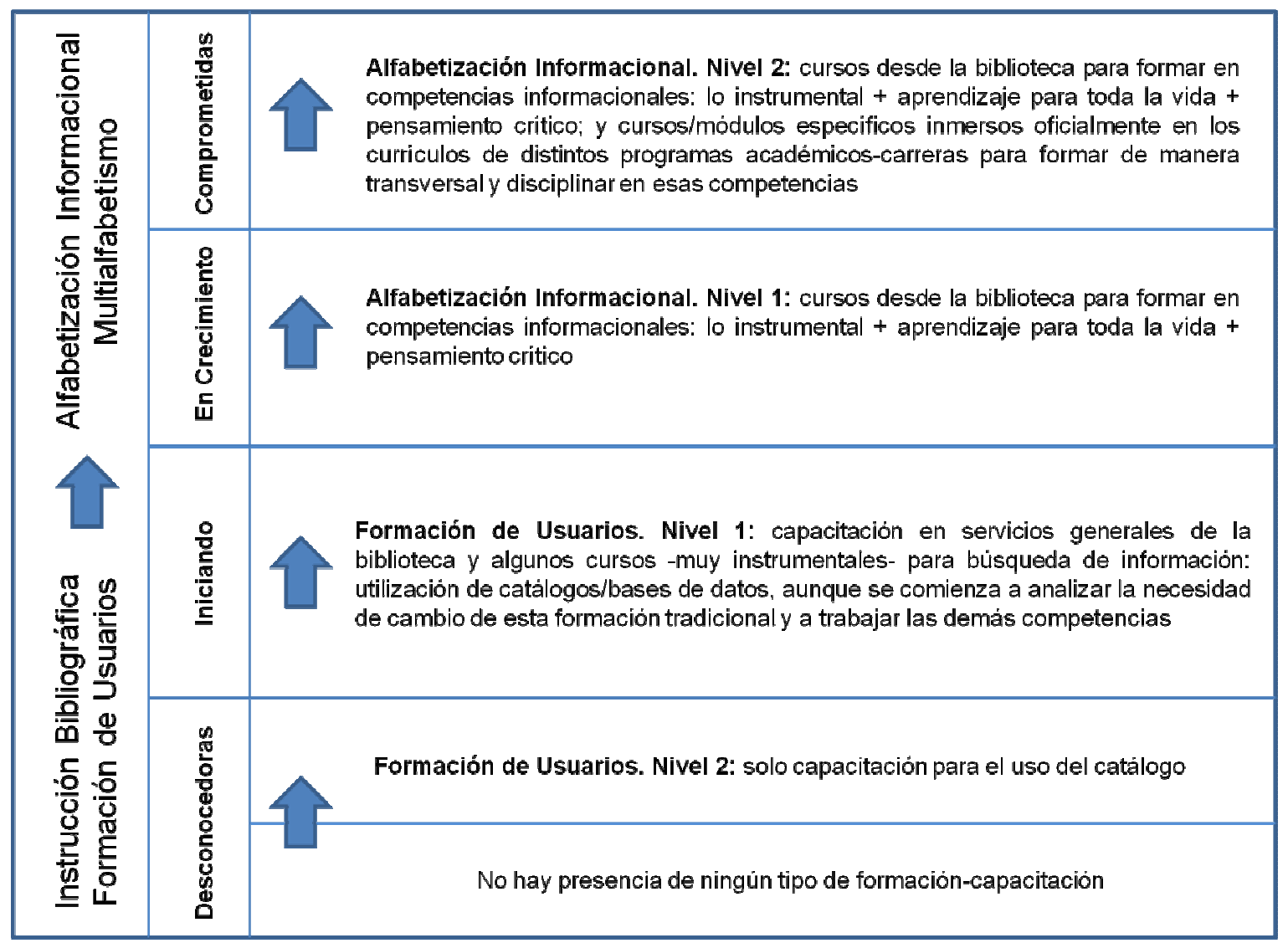

Figura 1. Niveles de incorporación de ALFIN-COMPINFO en universidades.

Es importante indicar al tener en cuenta este marco metodológico y teórico-conceptual para visualizar los posibles niveles de incorporación de ALFIN-COMPINFO en las bibliotecas-CRAI universitarias, como se indicó particularmente en cada uno de los análisis por país y sus respectivas publicaciones, que esta categorización es una aproximación desde la información que conllevan los sitios web, pero en ningún momento implica que categorizar una u otra universidad-IES / Dependencia en alguna de esas 4 categorías sea su total realidad en esta temática, ya que: "no siempre en los sitios web se publica-informa todo lo que se hace" o por el contrario "se publica-informa más de lo que realmente se hace"; por tanto, este tipo de análisis es una fotografía en un momento y desde un ángulo determinado que lleva a una aproximación y sistematización útil como visión general del desarrollo que se estaría dando en la formación en ALFIN-COMPINFO.

\section{MÉTODO.}

Para la realización del análisis de cada país se siguió una metodología semejante para cada caso. En primer lugar, se ubicaban los sitios web oficiales relativos a universidades, a educación superior, en los cuales se tuviera referencia y/o acceso directo a los listados de universidades-IES de dicho país y a sus sitios web.

En segundo lugar, para depurar y ampliar esos listados gubernamentales se recurrió a dos fuentes internacionales que posibilitan la identificación y el acceso a la dirección web de cada universidad: 
- Ranking web de universidades del mundo del CCHS/CSIC: $<$ http://www.webometrics.info/es $>$

- Portal estudiantil: El Altillo.com: <http://www.altillo.com/universidades/index.asp>

Esta metodología permitió generar una base de datos para cada país de sus universidades-IES, y específicamente de sus bibliotecas a analizar (públicas o privadas/particulares) y sus respectivos sitios web (url's):

\begin{tabular}{|l|c|c|c|c|}
\hline \multicolumn{1}{|c|}{ PAÍ́ } & $\begin{array}{c}\mathbf{N}^{\mathbf{0}} \text { DE } \\
\text { UNIVERSIDADES-IES }\end{array}$ & PÚBLICAS & $\begin{array}{c}\text { PRIVADAS/ } \\
\text { PARTICULARES }\end{array}$ & $\begin{array}{c}\text { PORCENTAJE DE } \\
\text { CADA PAÍS EN EL } \\
\text { ANÁLISIS }\end{array}$ \\
\hline Argentina & 131 & 66 & 65 & $4.8 \%$ \\
\hline Bolivia & 69 & 16 & 53 & $2.5 \%$ \\
\hline Brasil & 465 & 224 & 241 & $17 \%$ \\
\hline Chile & 79 & 21 & 58 & $2.9 \%$ \\
\hline Colombia & 337 & 106 & 231 & $2.3 \%$ \\
\hline Costa Rica & 75 & 19 & 56 & $1.3 \%$ \\
\hline Cuba & 36 & 36 & 0 & $3 \%$ \\
\hline Ecuador & 82 & 30 & 52 & $1.8 \%$ \\
\hline El Salvador & 49 & 4 & 45 & $4.8 \%$ \\
\hline España & 131 & 50 & 81 & $2 \%$ \\
\hline Guatemala & 54 & 10 & 44 & $1.4 \%$ \\
\hline Honduras & 38 & 12 & 26 & $3.4 \%$ \\
\hline México & 532 & 260 & 272 & $2.1 \%$ \\
\hline Nicaragua & 103 & 33 & 70 & $2.2 \%$ \\
\hline Panamá & 57 & 20 & 37 & $3.9 \%$ \\
\hline Paraguay & 59 & 6 & 53 & $3 \%$ \\
\hline Perú & 106 & 36 & 70 & $1.8 \%$ \\
\hline Portugal & 82 & 39 & 43 & $1.8 \%$ \\
\hline Puerto Rico & 48 & 10 & 38 & $1.5 \%$ \\
\hline Rep. Dominicana & 48 & 2 & 46 & $4.2 \%$ \\
\hline Uruguay & 41 & 14 & 27 & \\
\hline Venezuela & 114 & 67 & 47 & \\
\hline \multicolumn{1}{|c|}{ TOTALES } & $\mathbf{2 7 3 6}$ & $\mathbf{1 0 8 1}$ & $\mathbf{1 6 5 5}$ & \\
\hline
\end{tabular}

Tabla I. Sitios web analizados de bibliotecas de universidades-IES iberoamericanas.

Para la realización del análisis de estas 2736 bibliotecas de universidades-IES iberoamericanas se utilizó una ficha considerando postulados del análisis de contenidos (White y Marsh, 2006), en la cual se registraban los datos básicos de cada biblioteca, su url, la información sobre ALFIN-COMPINFO teniendo en cuenta las 7 variables a valorar, especialmente la de Estructuración del programa, y finalmente considerando esa información, en cuál de los 4 niveles de incorporación de ALFIN-COMPINFO se encontrarían.

\section{ANÁLISIS Y RESULTADOS.}

Considerando entonces ese total de 2736 sitios web de bibliotecas universitarias analizadas, e integrando los resultados de los 22 países iberoamericanos, encontramos lo siguiente respecto a los niveles de incorporación de ALFIN-COMPINFO (Figura 1) que dan cuenta de una imagen de la realidad de esta temática en este contexto se obtiene la Tabla II.

Teniendo en cuenta estos datos y los cruces de variables posibles entre países, universidades de carácter público o privado/particular y los niveles de incorporación de ALFIN-COMPINFO (Figura 1), podemos presentar dos tipos de análisis, uno más general y uno más específico. 


\begin{tabular}{|c|c|c|c|c|c|}
\hline PAÍS & AI2 & AI1 & FU1 & FU2 & $\begin{array}{c}\text { NO } \\
\text { PRESENTA } \\
\end{array}$ \\
\hline Argentina & 1 & 9 & 20 & 40 & 61 \\
\hline Bolivia & 0 & 0 & 1 & 12 & 56 \\
\hline Brasil & 3 & 30 & 95 & 121 & 216 \\
\hline Chile & 2 & 11 & 9 & 23 & 34 \\
\hline Colombia & 5 & 11 & 22 & 37 & 262 \\
\hline Costa Rica & 2 & 3 & 9 & 13 & 48 \\
\hline Cuba & 4 & 8 & 12 & 4 & 8 \\
\hline Ecuador & 0 & 0 & 9 & 10 & 63 \\
\hline El Salvador & 0 & 1 & 5 & 16 & 27 \\
\hline España & 10 & 19 & 29 & 18 & 55 \\
\hline Guatemala & 0 & 0 & 2 & 15 & 37 \\
\hline Honduras & 0 & 3 & 1 & 9 & 25 \\
\hline México & 5 & 15 & 18 & 34 & 460 \\
\hline Nicaragua & 0 & 0 & 13 & 3 & 87 \\
\hline Panamá & 0 & 0 & 8 & 5 & 44 \\
\hline Paraguay & 0 & 0 & 3 & 6 & 50 \\
\hline Perú & 0 & 5 & 8 & 13 & 80 \\
\hline Portugal & 2 & 5 & 19 & 11 & 45 \\
\hline Puerto Rico & 3 & 9 & 8 & 4 & 24 \\
\hline Rep. Dominicana & 0 & 0 & 8 & 9 & 31 \\
\hline Uruguay & 0 & 0 & 10 & 3 & 28 \\
\hline Venezuela & 0 & 5 & 2 & 15 & 92 \\
\hline TOTALES & 37 & 134 & 311 & 421 & 1833 \\
\hline
\end{tabular}

Tabla II. Total de bibliotecas universitarias analizadas y niveles de incorporación por país.

Lo anterior implica que solo un 30\% (903 bibliotecas-instituciones) de ese total de las bibliotecas universitarias iberoamericanas, según la información de sus sitios web, sí tendría la formación como un servicio o acción estratégica. Cuando se indaga en este 30\% considerando las 4 categorías de incorporación que se proponen desde esta investigación (Figura 1) encontramos los siguientes resultados porcentualmente:

- Un $46.6 \%$ (equivalente al $15.4 \%$ del total de bibliotecas analizadas) estaría realizando acciones de formación del tipo de FU1 (421 bibliotecas-instituciones) y un 34.4\% (equivalente al 11.3\% del total de bibliotecas analizadas) del tipo FU2 (311 bibliotecas-instituciones), es decir, integrando estos porcentajes, podemos indicar que el $81 \%$ (732 bibliotecas instituciones) aún está desarrollando una formación de usuarios muy tradicional.

- Por tanto, el $19 \%$ de esas 903 bibliotecas-instituciones (equivalente al $6.2 \%$ del total de bibliotecas analizadas) serían solo las bibliotecas-instituciones iberoamericanas que sí estarían trabajando a nivel de ALFINCOMPINFO, lo cual equivaldría en síntesis, que del total de 2736 bibliotecas de universidades-IES iberoamericanas solo 171 estarían realizando servicios-acciones de alfabetización informacional-competencias informacionales, según la información que presentan en sus sitios web. De estas, 135 (equivalente al $4.9 \%$ del total de bibliotecas-instituciones iberoamericanas) estarían en el nivel AI1 y solo 36 (equivalente al 1.3\% del total de bibliotecas-instituciones iberoamericanas) estarían en el nivel de AI2, que es el relativo a la ALFINCOMPINFO como parte curricular (con cursos concretos y notas válidas para la hoja de vida estudiantil) dentro de determinados programas académicos o en toda una universidad-IES. 
A su vez, si consideramos los resultados anteriores, y tenemos en cuenta la variable de tipología de universidadIES sea pública o privada/particular (Tablas III y IV), se encuentra que:

- De las 1833 bibliotecas-instituciones que NO presentan en sus sitios web la formación como servicio o acción estratégica, 582 son de carácter público y 1251 de carácter privado/particular. Porcentualmente, estos datos equivalen a que del total de bibliotecas-instituciones SIN presencia de formación, el 31.7\% son públicas y el $68.3 \%$ son privadas, lo cual muestra una desafortunada tendencia que desde otros ámbitos se ha criticado: la privatización y/o mercantilización de la educación superior dando paso en muchos casos a instituciones privadas/particulares de baja calidad, en las que solo hay una docencia básica, si investigación, y donde la biblioteca brilla por su ausencia (no había ninguna referencia-link desde el portal de la universidad) o es solo un link para acceder al catálogo pero sin servicios e información de los mismos, dando cuenta de una concepción de biblioteca más como "repositorio-almacén de libros".

- De las 903 bibliotecas-instituciones que SÍ tienen la formación de sus usuarios como servicio o acción estratégica (FU2 + FU1 + AI1 + AI2): 500 son de carácter público (equivalente al 53.4\%) y 403 (equivalente al $44.6 \%$ ) de carácter privado/particular, lo cual es proporcionalmente más parejo que entre las que NO presentan la formación. No obstante, cuando se profundiza entre éstas, en los 4 niveles de incorporación (Figura 1), se encuentra que en la generalidad, son las bibliotecas-instituciones públicas las que más han avanzado en ALFIN-COMPINFO comparativamente con las privadas/particulares: bibliotecas-instituciones públicas con presencia de formación FU2 y FU1: 381 y con presencia de formación AI1 y AI2: 119; mientras que bibliotecas-instituciones privadas/particulares con presencia de formación FU2 y FU1: 351 y con presencia de formación AI1 y AI2: 52.

\subsection{Análisis y resultados específicos.}

De manera específica, centrándonos en los resultados referentes a los niveles AI1 y AI2 (las 171 bibliotecasinstituciones) que son los que representan efectivamente la incorporación de la ALFIN-COMPINFO en las universidades-IES iberoamericanas desde el accionar de sus bibliotecas y la información que presentan sus sitios web, y considerando las variables de país y carácter de institución (pública o privada/particular); podemos resaltar los siguientes aspectos:

- La mayor cantidad de bibliotecas-instituciones identificadas en estos dos niveles estarían en 12 de los 22 países iberoamericanos (167 de las 171 bibliotecas-instituciones identificadas). En su orden serían: Brasil con 33; España con 29; México con 20; Cuba con 16; Chile con 13; Colombia con 12; Puerto Rico con 12; Argentina con 10; Portugal con 7; Costa Rica con 5; Perú con 5 y Venezuela con 5.

- Y entre estas, la mayoría están en bibliotecas-instituciones públicas en los diferentes niveles AI1 y AI2:

\begin{tabular}{|c|c|c|c|c|c|c|c|}
\hline PAÍS & PÚBLICA & PRIVADA/PART. & AI1 & PÚBLICA & PRIVADA/PART. & AI2 & SUBTOTAL \\
\hline 1. Brasil & 25 & 5 & 30 & 3 & 0 & 3 & 33 \\
\hline 2. España & 18 & 1 & 19 & 9 & 1 & 10 & 29 \\
\hline 3. México & 9 & 6 & 15 & 5 & 0 & 5 & 20 \\
\hline 4. Cuba & 12 & 0 & 12 & 4 & 0 & 4 & 16 \\
\hline 5. Chile & 5 & 6 & 11 & 1 & 1 & 2 & 13 \\
\hline 6. Colombia & 2 & 6 & 8 & 1 & 3 & 4 & 12 \\
\hline 7. Puerto Rico & 3 & 6 & 9 & 3 & 0 & 3 & 12 \\
\hline 8. Argentina & 1 & 8 & 9 & 1 & 0 & 1 & 10 \\
\hline 9. Portugal & 5 & 0 & 5 & 1 & 1 & 2 & 7 \\
\hline 10. Costa Rica & 3 & 0 & 3 & 2 & 0 & 2 & 5 \\
\hline 11. Perú & 1 & 4 & 5 & 0 & 0 & 0 & 5 \\
\hline 12. Venezuela & 4 & 1 & 5 & 0 & 0 & 0 & 5 \\
\hline Totales & 88 & 43 & 131 & 30 & 6 & 36 & 167 \\
\hline
\end{tabular}

Tabla III. Distribución según carácter de público o privadas/particulares de la mayor cantidad de bibliotecas universitarias iberoamericanas según país en niveles AI1 y AI2. 
- No obstante, cuando se analiza esta presencia de niveles AI1 y AI2 desde la perspectiva porcentual, según el número de bibliotecas-instituciones universitarias iberoamericanas, se visualiza que este orden varía en forma considerable:

\begin{tabular}{|l|c|c|c|c|c|}
\hline \multicolumn{1}{|c|}{ PAÍS } & AI1 & AI2 & SUBTOTAL & $\begin{array}{c}\text { N TOTAL DE } \\
\text { BIBLIOTECAS } \\
\text { UNIVERSITARIAS }\end{array}$ & $\begin{array}{c}\text { PORCENTAJE DE BIBLIOTECAS } \\
\text { EN NIVELES DE AI1 Y AI2 DEL } \\
\text { TOTAL NACIONAL }\end{array}$ \\
\hline 1. Cuba & 12 & 4 & $\mathbf{1 6}$ & 36 & $\mathbf{4 4 , 4 \%}$ \\
\hline 2. Puerto Rico & 9 & 3 & $\mathbf{1 2}$ & 48 & $\mathbf{2 5 , 0 \%}$ \\
\hline 3. España & 19 & 10 & $\mathbf{2 9}$ & 131 & $\mathbf{2 2 , 1 \%}$ \\
\hline 4. Chile & 11 & 2 & $\mathbf{1 3}$ & 79 & $\mathbf{1 6 , 5 \%}$ \\
\hline 5. Portugal & 5 & 2 & $\mathbf{7}$ & 82 & $\mathbf{8 , 5 \%}$ \\
\hline 6. Argentina & 9 & 1 & $\mathbf{1 0}$ & 131 & $\mathbf{7 , 6 \%}$ \\
\hline 7. Brasil & 30 & 3 & $\mathbf{3 3}$ & 465 & $\mathbf{7 , 1 \%}$ \\
\hline 8. Costa Rica & 3 & 2 & $\mathbf{5}$ & 75 & $\mathbf{6 , 7 \%}$ \\
\hline 9. Perú & 5 & 0 & $\mathbf{5}$ & 106 & $\mathbf{4 , 7 \%}$ \\
\hline 10. Venezuela & 5 & 0 & $\mathbf{5}$ & 114 & $\mathbf{4 , 4 \%}$ \\
\hline 11. México & 15 & 5 & $\mathbf{2 0}$ & 532 & $\mathbf{3 , 8 \%}$ \\
\hline 12. Colombia & 8 & 4 & $\mathbf{1 2}$ & 337 & $\mathbf{3 , 6 \%}$ \\
\hline Totales & $\mathbf{1 3 1}$ & $\mathbf{3 6}$ & $\mathbf{1 6 7}$ & $\mathbf{2 1 3 6}$ & \multicolumn{2}{|c|}{} \\
\hline
\end{tabular}

Tabla IV. Porcentaje de incorporación de ALFIN-COMPINFO (AI1 y AI2) entre bibliotecas-instituciones universitarias iberoamericanas según la información de sus sitios web.

\section{CONCLUSIONES Y RECOMENDACIONES.}

Teniendo en cuenta los resultados generales y específicos presentados en los ítems anteriores este texto presenta las siguientes conclusiones:

- La primera conclusión es que en lo referente a la incorporación de ALFIN-COMPINFO aún hay mucho trabajo por hacer para lograr que esta temática sea más estratégica al interior de las mismas bibliotecas (un 70\% NO presenta información sobre servicios-acciones de formación), y desde estas, o con el apoyo de estas, en las universidades y la sociedad en general, para así lograr una ciudadanía alfabetizada-competente informacionalmente como lo viene insistiendo la UNESCO desde diferentes eventos y declaraciones (UNESCO, 2003, 2006, 2010, 2011, 2012) ${ }^{2}$, desde una perspectiva integral-holística de ALFIN-COMPINFO en relación con otras alfabetizaciones-competencias (multialfabetismo).

- Por otro lado, es necesario que las bibliotecas que sí reconocen la formación, pero que desde años atrás llevan realizando un trabajo tradicional o muy centrado en las dos primeras subcompetencias informacionales: búsqueda y localización de la información (un $81 \%$ de las que tienen servicios o realizan acciones de formación), amplíen su perspectiva y gestionen recursos proactivamente para así dar el paso de la formación de usuarios tradicional a la ALFIN-COMPINFO no solo ampliando contenidos formativos sino asumiendo posturas y metodologías que conllevan a la afirmación de la ALFIN-COMPINFO como facilitadora del aprendizaje para toda la vida y evita la brecha digital, informacional y social (Pinto Molina y Uribe Tirado, 2011).

- En relación con lo anterior, es importante conocer y reconocer el trabajo que vienen realizando las 171 bibliotecas universitarias que presentan servicios-acciones de ALFIN-COMPINFO, en las que asumen trabajar casi todas o todas las 6 o 7 subcompetencias informacionales, para con ello mirar sus procesos, cómo llegaron a esos niveles, sus lecciones aprendidas, etc.; pues son ellas las universidades-IES líderes en el contexto iberoamericano ${ }^{3}$, donde insistimos en la conclusión que aún hay mucho por hacer si consideramos, que estas 171 bibliotecas-instituciones solo equivalen al 6.2\% del total de universidades-IES iberoamericanas. 
- A su vez, una conclusión específica implica el considerar también la variable de universidad-IES públicas o privadas/particulares, ya que como se mostró en los resultados, hay en términos generales un mayor avance y conciencia de la importancia de la formación en lo informacional entre las universidades-IES de carácter público (el 69.9\% de todas las de niveles AI1 o AI2), sobre todo en determinados países (Brasil, España, México, Cuba, Portugal, Costa Rica y Venezuela), y por tanto, un mayor trabajo por hacer en las universidades privadas/particulares. No obstante, es importante reconocer que en algunos otros países (Argentina, Colombia y Perú) esta relación es a la inversa. En síntesis, estos niveles de desarrollo tienen que ver directamente con las realidades de apoyo institucional, presupuestos, capital humano y apertura a innovaciones tecnológicas y pedagógicas, sean universidades-IES públicas o privadas/particulares.

- Por otro lado, considerando la relación número de universidades-IES con niveles de AI1 ó AI2 y el total de universidades-IES, es evidente que determinados países aunque son los de mayores casos en términos de cantidad, porcentualmente tienen menores niveles de incorporación teniendo en cuenta todas las universidadesIES de su país (Brasil, México, Colombia), y al contrario, no siendo el país que aporta más casos, sí presentar un alto porcentaje de incorporación (Cuba, Puerto Rico). A su vez, visualizar la realidad de otros países que reportan similares niveles de: cantidad de casos identificados y porcentaje de incorporación (España, Chile, Argentina, Costa Rica, Perú, Venezuela).

Y finalmente, una última conclusión, relativa más con lo metodológico e investigaciones futuras:

- La posibilidad de realizar estudios que abarquen todo el contexto iberoamericano a nivel universitario y de bibliotecas, y el aprovechar fuentes de información que permiten la recopilación y acceso a información de este tipo. En el caso de esta investigación queda una base de datos de 2736 universidad-IES iberoamericanas (sin ser pretenciosos, podría decirse que están casi la totalidad) que permitirá seguir con esta línea de investigación, mirando en el caso de ALFIN-COMPINFO sus avances y desarrollo a mediano o largo plazo en unos años, o aprovechar la misma y el acceso a los link de estas bibliotecas universitarias, para hacer otras investigaciones sobre otros servicios, sobre sus portales y su accesabilidad o usabilidad, etc.

A su vez, estos resultados generales y específicos, y estas conclusiones, reafirman varias recomendaciones expresadas desde la Declaración de la Habana (2012) que queremos resaltar como llamado final desde este texto, ante la necesidad de un trabajo colaborativo por la ALFIN-COMPINFO en nuestro contexto iberoamericano (o latinoamericano según se quiera asumir):

- Generar espacios para el intercambio continuo de los aprendizajes adquiridos en el desarrollo de los programas de formación en los diferentes contextos: coordinar la realización periódica de eventos académicosprofesionales en los diferentes países, que busquen la participación de coordinadores, formadores y líderes de ALFIN locales, nacionales e internacionales (iberoamericanos y de otros contextos), con el objetivo de fundar un espacio continuo de intercambio de experiencias, lecciones aprendidas y generación de contactos para trabajos comunes entre profesionales, bibliotecas, instituciones educativas y distintas organizaciones en cada país o entre distintos países.

- Apoyar y apoyarnos mutuamente en el crecimiento y desarrollo de los programas formativos: reconocer los avances alcanzados en el desarrollo de la ALFIN en las propias bibliotecas, instituciones educativas u organizaciones, y que por tanto, se tiene también la posibilidad de apoyar a aquellas otras instituciones del contexto local y nacional, e incluso internacional (especialmente de Iberoamérica) que quieran avanzar en ALFIN pero cuyos procesos en el tema son aún muy incipientes. La orientación-formación, las lecciones aprendidas y el compartir recursos-objetos de aprendizaje son acciones fundamentales para que puedan avanzar más rápidamente en el desarrollo e implementación de sus propios programas de ALFIN. A su vez, estas acciones constituyen, para las instituciones más avanzadas, una posibilidad de seguir creciendo desde una perspectiva de mejoramiento continuo, gracias a la colaboración y generación de redes.

- Facilitar y mantener el intercambio y apoyo mediante el uso de distintos recursos web: promover la sostenibilidad y la creación de nuevos recursos web 1.0, 2.0 o 3.0 sobre ALFIN (portales, blogs, wikis, listas de discusión, comunidades virtuales, herramientas de web semántica, etc.) en cada país o en diferentes bibliotecas, instituciones educativas u organizaciones que fomenten la ALFIN en sus contextos; y apoyar e integrar dichos recursos, que gestionan distintos profesionales u organizaciones en Iberoamérica, para conocer más a fondo el desarrollo en cada contexto, y de la región en general, y de esa manera, dar cabida a mayores posibilidades de trabajo colaborativo. 
- Generar temáticas de investigación pertinentes y que fomenten el trabajo colaborativo interdisciplinario y transdisciplinario: establecer temáticas de investigación aplicada que apoyen el crecimiento, desarrollo y evaluación de la ALFIN en diferentes contextos, que consideren los diversos niveles y formas educativas en las que se esté llevando a la práctica esta formación, para que se favorezca el trabajo conjunto entre profesorescapacitadores, catedráticos-investigadores, bibliotecólogos-bibliotecarios, y demás personas que puedan apoyar este proceso.

- Realizar y generar trabajos conjuntos para lograr diagnósticos actualizados sobre el desarrollo de esta formación en cada contexto: fomentar y facilitar el acceso a los insumos documentales, de contenidos y metodológicos que posibiliten la realización de diagnósticos y estados del arte en el desarrollo de la ALFIN en contextos institucionales, locales, nacionales y/o internacionales (especialmente iberoamericanos) sobre esta temática; y que permitan identificar y evaluar niveles de desarrollo y líneas de acción para avanzar en el tema, y formas de aprendizaje y adaptación de las experiencias de otros contextos y maneras de trabajo conjunto.

\section{NOTAS.}

${ }^{1}$ Ferreira (1995); Gómez Hernández (2000); Menou (2004); Rader (2002); Dudziak (2002, 2003, 2008); Gómez Hernández, Pasadas Ureña (2003); Campello (2003); Angulo Marcial (2003); Dudziak, Ferreira (2004); Vives I Gràcia (2004); Lecardelli, Schoffen (2006); Fernandez Aballi/Unesco (2007); Lau et al. (2007); Marzal, Calzada Prado (2007); Pinto, Sales (2007); Da Rocha et al. (2008); Area Moreira, Gros, Marzal (2008); Meneses Placeres (2009); Licea de Arenas (2009); Da Silva et al. (2009, 2010); Pinto, Cordón y Gómez (2010) y Uribe Tirado (2010, 2011, 2012), entre otros.

${ }^{2}$ Ver las referencias a estas 13 publicaciones en el Anexo 1: <http://alfiniberoamerica.blogspot.com/2012/06/analisis-de-alfin-para-lasbibliotecas.html>.

${ }^{3}$ Este resultado coincide indirectamente con el estado del arte de esta temática a nivel iberoamericano, ya que al realizar un análisis de contenidos sobre esta literatura y los casos que son reportados en la misma de bibliotecas-instituciones universitarias iberoamericanas que tienen la ALFINCOMPINFO como servicio, que están realizando acciones o que tienen un programa de formación estructurado, son estas mismas bibliotecasinstituciones las que aparecen reportadas. Ver estado del arte de ALFIN-COMPINFO en Iberoamérica en: $<$ http://alfiniberoamerica.wikispaces.com $>$.

\section{BIBLIOGRAFÍA.}

Declaración de La Habana 15 ACCIONES de ALFIN...por un trabajo colaborativo y de generación de redes para el crecimiento de la alfabetización informacional en el contexto de los países iberoamericanos. En: Congreso INFO Cuba, Seminario ALFIN / Iberoamérica. 2012. <http://alfiniberoamerica.blogspot.com>. [Consulta: 19 de abril de 2012].

DETLOR, B. y LEWIS, V. Academic Library Web Sites: Current Practice and Future Directions. The Journal of Academic Librarianship, 2006, vol. 32, nº 3, p. 251-258.

EMPATIC. Report on Current State and Best Practices in Information Literacy. European Commission: Lifelong Learning Programme, 2009. <http://empat-ic.eu/eng/Reports/D1.1-Report-on-Current-State-and-Best-Practices-inInformation-Literacy-Final>. [Consulta: 11 de diciembre de 2012].

FERREIRA-GOMES, H.; PRUDÊNCIO, D.S. y DA CONCEIÇÃO, A.V. A mediação da informação pelas Bibliotecas universitárias: um mapeamento sobre o uso dos dispositivos de comunicação na web. Informação \& Sociedade: Estudos, 2010, vol. 20, $\mathrm{n}^{\mathrm{o}} \quad 3, \quad$ p. $1-12$. Disponible en: $<$ http://www.ies.ufpb.br/ojs2/index.php/ies/article/view/9047/4812>. [Consulta: 9 de enero de 2011].

GARDNER, S.J; JURICEK, J.E. y XU, F.G. An Analysis of Academic Library Web Pages for Faculty. The Journal of Academic Librarianship, 2008, vol. 34, no 1, p. 16-24.

PINTO MOLINA, M. y URIBE TIRADO, A. Formación del bibliotecario como alfabetizador informacional. Anuario ThinkEPI, 2011. Disponible en: <http://www.thinkepi.net/anuario-thinkepi/anuario-thinkepi-2011>. [Consulta: 29 de abril de 2011].

SONNTAG, G. Information literacy classification-Colombia. Facilitating Information Literacy Education. 2011

Disponible en: $<$ http://www.linkedin.com/groupItem?view=\&gid=1850160\&type=member\&item=48506240\&qid=b59f064e713d-4286-b98d-fe572337839f\&trk=group_most_popular-0-b-ttl\&goback=\% 2Egmp_1850160>. [Consulta: 27 de marzo de 2011].

URIBE TIRADO, A. Avances y perspectivas de ALFIN en Iberoamérica. Una mirada desde la publicación académico-científica y la web 1.0 y 2.0. Congreso INFO Cuba 2010 - Instituto de Información Científica y Tecnológica (IDICT), 2010a, p. 1-30. Disponible en: <http://eprints.rclis.org/handle/10760/14638>. [Consulta: 21 de febrero de 2011].

URIBE TIRADO, A. La Alfabetización Informacional en Iberoamérica. Una aproximación a su pasado, presente y futuro desde el análisis de la literatura publicada y los recursos web. IBERSID. Revista de sistemas de información 
y documentación. Universidad de Zaragoza. 2010, p. 165-176. Disponible en: $<$ http://eprints.rclis.org/handle/10760/14638>. [Consulta: 21 de febrero de 2011].

URIBE TIRADO, A. La Alfabetización Informacional en la Universidad. Descripción y Categorización según los Niveles de Integración de ALFIN. Caso Universidad de Antioquia. Revista Interamericana de Bibliotecología,

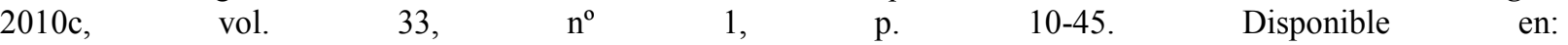
$<$ http://aprendeenlinea.udea.edu.co/revistas/index.php/RIB/article/view/6280/5801>. [Consulta: 21 de febrero de 2011].

URIBE TIRADO, A. y MACHETT'S PENAGOS, L.E. Informe-Estado del Arte de la Alfabetización Informacional en Colombia. IFLA - Information Literacy Section. Marzo 2011, p. 26. Disponible en: $<$ http://www.ifla.org/en/publications/information-literacy-state-of-the-art-report-colombia-espa-ol>. [Consulta: 21 de febrero de 2011].

WEBBER, S. y JOHNSTON, B. "Working towards the information literate university". En: Walton, G. y Pope, A. (Eds.). Information literacy: recognising the need. Staffordshire University, Stoke-on-Trent. Oxford: Chandos, 2006, p. 47-58. Disponible en: <http://dis.shef.ac.uk/sheila/staffs-webber-johnston.pdf>. [Consulta: 20 de octubre de 2009].

WANG, L. Sociocultural learning theories and information literacy teaching activities in higher education. Reference \& User Services Quarterly, 2007, vol. 47, $\mathrm{n}^{\mathrm{o}}$ 2, p. 149-158. Disponible en: <http://www.bui.hawhamburg.de/pers/ursula.schulz/teaching_library_2/wang_2007.pdf $>$. [Consulta: 11 de diciembre de 2012].

WHITE, M.D. y MARSH, E.E. Content Analysis: A Flexible Methodology. Library Trends, 2006, vol. 55, nº 1, p. 2245. 